\title{
Foreign reports
}

\section{Psychiatry in Saudi Arabia: an overview}

\author{
A. Al-Sabaie, Clinical Fellow in Psychiatry, Ottawa University, Ottawa, Ontario, \\ Canada
}

The population of Saudi Arabia is about 10 million, $37 \%$ of whom live in the cities. Birth control is discouraged because of cultural and religious beliefs and the average size of the family unit is six people. Islam is the religion of the state and it is the source of its civil and criminal legislative systems. As a divine law it cannot be revoked in most instances.

\section{Mental health services}

Health services, including medications, are provided free, for the most part by the Ministry of Health and to a lesser extent by other government hospitals. Traditional medicine is practised mainly in the rural areas, where the nomadic and traditional people live. Witchcraft, cautery, divine rituals, exorcism and beating (of psychotic patients who are thought to be possessed) are used for treatment. Many psychiatric patients seek help from traditional healers before turning to hospitals. Most of the mental hospital beds are in a large mental hospital, Shehar, founded in 1962 in Taif. The rest are in short-stay mental hospitals or in general hospital psychiatry units.

\section{Teaching and practice of psychiatry}

Psychiatry is taught to medical students in weekly two or three hour sessions in their last two years. In two of the four available medical schools where full psychiatric services are lacking, students are sent to a psychiatric hospital for an intensive four week course. Graduate training in psychiatry is available at King Saud University in Riyadh in a two year diploma course. However, for consultant posts, the British MRCPsych or an equivalent degree is required. Admission is usually negotiated between patients, their family and the psychiatrist, as there is no Mental Health Act system to control the need for and the length of hospital stay. The practice is medication-oriented and psychotherapy when practised is brief and supportive. Day hospitals are rare and halfway houses are not available, as cultural and religious beliefs emphasise strong family ties and social support. Almost all the consultant psychiatrists and nursing staff are non-Saudis. Most of the psy- chiatrists are Arabic/English speaking, while most of the nursing staff are westerners, speaking only English.

\section{Epidemiology and clinical patterns of mental illness}

There is a severe deficiency in epidemiological studies of mental disorders in Saudi Arabia. A few recent service utilisation studies shared some interesting findings (El-Sayed et al, 1986; El-Rufaie, 1988). The striking finding that men outnumber women was felt to be more apparent than real, since women may not have the same degree of access to hospitals. For cultural reasons women are dependent on men to take them to hospital. Moreover, women in Eastern societies are more tolerant of both physical and psychological pain which may be considered normative for them (El-Sayed, 1986). An alternative explanation is the protective role of the extended Saudi family. Most of the psychiatric service users were in their second or third decade of life; this is felt to be due to the multiple stresses and conflicts between traditional and modern values brought about by rapid urbanisation in the country (El-Sayed, 1986). This is similar to the findings of El-Islam (1986) in other Arab gulf states and Mehrayar \& Tashakkori (1978) in Iran of an inter-generational conflict involving patterns of family relations, methods of marriage and the role of women in society. The small representation of the elderly could be explained by their shorter life span in this developing country and by the Islamic teachings ingrained in Saudi culture regarding absolute respect and care for old people, who may even be considered a blessing. Hysteria appears to be quite prevalent in Saudi Arabia in keeping with its high occurrence in developing societies.

Chaleby (1987a) found social phobia to be quite prevalent in Saudis, especially among young unmarried males with relatively higher educational and occupational levels. This finding may not be true for the general Saudi population since the King Faisal Specialist Hospital is a referral hospital available only to a special population. The findings were related to the rigid and highly valued customs and 
rituals involving every social situation in Saudi culture. The low incidence of this disorder in women was felt to be because Saudi women are protected from exposure to anxiety-provoking situations as their gatherings are mostly recreational with minimal rituals.

Schizophrenia seems to cluster in families in Saudi Arabia due to consanguinity (Chaleby, 1987b). As expected in developing countries, catatonic states are common; this may be explained partly by ethnicity, the small number of psychiatrists and the public's ignorance, which may lead to delay in seeking help until the illness becomes life-threatening. Similar to Govindasamy's finding in India (Govindasamy, 1958), mutism, stupor and psychomotor retardation are more common presentations than excitement.

There is no mention of eating disorders in Saudi Arabia in the psychiatric literature. This perhaps reflects the rarity of these disorders there, due to the role of a culture that idealises modesty and discourages public display of the female figure. In fact, mild obesity is considered by many as a sign of health. In contrast to the findings of Murphy et al, 1963, guilt feelings are uncommon compared to the West, reflecting the Islamic view of sin, penitence and forgiveness. Affective psychosis was the most common reason for admission and depression was most prevalent in middle-aged housewives.

Suicidal behaviour and deliberate self harm are rare and most suicide attempters are young, single females impulsively overdosing after an interpersonal conflict. These findings contrast with those of Daradkeh \& Al-Zayer (1988) although the sample of this study is representative of the Saudi population. Asking about death wishes is about the only way to assess a potentially suicidal patient without offending him, since suicide is considered an unforgivable sin.

Alcohol and drug abuse are relatively uncommon and this could be explained by the strong Islamic attitude and the strict legislation of the country, which has recently passed a law making death the punishment of drug traffickers.

Violent destructive behaviour, adultery and paraphilia are forbidden in Islamic law. These disorders are dealt with by the criminal code system, which may partly explain the under-representation of these disorders in psychiatric practice.

Male homosexuality is said to be common. However, Western observers would probably misinterpret socially acceptable modes of physical expression of warmth and friendship (such as holding hands and kissing in public) as homosexuality. Knowing that it exists in many cultures, in Saudi Arabia it is stigmatised by people, forbidden by religion and punishable by law. Nevertheless, it is recognised by mental health workers as an illness and treatment is offered for motivated patients.
Somatisation disorder is common, especially in lower social class patients and women, and although psychosomatic disorders are fairly common, they are seen (by both patients and physicians) as purely physical, sometimes ignoring their clear psychodynamic bases.

The Saudi family is a closed system with rigid boundaries, precluding therapeutic advantages of family or group therapy. This degree of cohesion and closeness of the extended family may provide protection of its members but it increases their sensitivity to disharmony in the large family system. Al-Khani et al (1986), for example, found a significantly higher rate of life events among Saudi married schizophrenic females compared to female controls or any other group in his study. This points to the special needs of this group for a more active community based social support system.

\section{Current problems and future trends}

There are few native Saudi consultant psychiatrists in the entire country, and despite financial incentives and availability of appropriate equipment there is a severe shortage of paramedical mental health staff.

Child psychiatry services are poorly developed in Saudi Arabia. The country has no more than two or three child psychiatrists but $44 \%$ of its population are under 15 years of age. Children are seldom seen in psychiatry, which is in part due to the public's and teachers' ignorance of children's feelings and behaviour, especially in younger age groups, and the complete dissociation between educational and health systems. Only severe types of children's mental illness are recognised and the sufferers offered special education in specialised schools, while the mildly or moderately disturbed may have to suffer the repeated failure and humiliation of going through the regular school system. Parents infrequently use physical methods of discipline; however, it is sometimes hard to draw a line between that and child abuse which is considered a punishable crime under Saudi Arabian law.

Individual efforts to standardise psychological measurements have been made in Saudi Arabia; however, collaboration with linguistics in an organised pattern is necessary to conduct any culturally valid, valuable research. Further studies on drug dose-responses are needed as suggested by Conacher et al (1987), who found a higher incidence of acute dystonic reactions in Saudi schizophrenic patients treated with haloperidol compared to Western patients.

Although there is no antipsychiatry movement, there are occasional pieces in daily newspapers about the "harmful effects" of medications and ECT 
(which is practised with the most modern methods and equipment). This is a very sensitive area for Saudi patients who consider inflicted self-harm a sin. For the overwhelming majority of patients, the therapist needs to demonstrate both knowledge and respect for their religious beliefs and values to be therapeutically effective. An example of this kind of problem is a patient insisting on fasting during the month of Ramadan (the fasting month for Moslems) while taking his lithium or else discontinuing it. The psychiatrist must demonstrate knowledge about general medicine and be able to provide concrete, practical discussion which are applicable to the kind of short-term treatment of patients that is familiar to the non-psychiatric physician. Females, as a rule, prefer a female therapist, whom they believe is more understanding and appreciative of women's problems. Luckily, there are more Saudi female physicians now than ever before.

Studies of traditional medicine as it pertains to psychiatry have just started as part of a big research project undertaken by King Saud University. Some liaison between physicians in private practice and health services in general is still lacking in Saudi Arabia. This increases the load on the already busy few psychiatrists and hence reduces the quality of follow-up. The important role of the extended family in providing support to its mentally ill members is made obvious by the high percentage of patients brought to emergency by their relatives and the reliance of the mental health system on their afterdischarge care.

Pasnau (1983) posed several questions regarding the implications for potential abuse of psychiatry, in the absence of mental health law and the possibility of developing new legal guidelines in the context of traditional Islamic law and customs in Saudi Arabia. This could be possible in the foreseeable future under the current Islamic resurgence movements and the trends of science Islamisation throughout the country.

\section{Acknowledgements}

I wish to thank Drs R. Chandrasena and V. F. Di Nicola for their continuing support and constructive criticism.

\section{References}

Al-Khani, M., Bebbington, P., Watson, J. \& House, F. (1986) Life events and schizophrenia: a Saudi Arabian study. British Journal of Psychiairy, 148, 12-22.

Chaleby, K. (1987a) Social phobia in Saudis. Social Psychiatry, 22, 167-170.

(1987b) Cousin marriages and schizophrenia in Saudi Arabia. British Journal of Psychiatry, 150, 547-549.

Conacher, G., Marazki, M. \& Evans, D. (1987) Acute dystonic reactions in Saudi Arabian psychiatric patients treated with haloperidol. Acta Psychiatrica Scandinavia, 75, 333-334.

DARADKeh, K. \& Al-ZAYER, N. (1988) Parasuicide in an Arab industrial community: The Arabian American Oil Company experience. Acta Psychiatrica Scandinavica, 77, 707-711.

El-Islam, M., Abu-Dagga, S., Malasi, T., Moussa, M. (1986) Inter-generational conflict and psychiatric syndrome. British Journal of Psychiatry, 149, 300-306.

El-Sayed, S., Maghraby, H., Hafeiz, H., Buckley, M. (1986) Psychiatric diagnostic categories in Saudi Arabia. Acta Psychiatrica Scandinavia, 74, 553-554.

GovindaSAMY, G. (1958) Cultural aspects of psychiatric illness. Journal of All India Institute of Mental Health, 1, 2.

MEHRAYAR, A. \& TAShakkori, G. (1978) Sex and parental education as determinants of marital aspirations and attitudes of a group of Iranian youth. Journal of Marriage and the Family, 40, 629-637.

MuRPhy, H., Wittakower, E. \& Chance, N. (1963) A cross-cultural inquiry into the symptomatology of depression. Transcultural Psychiatric Research Review, 1, 5-8.

Pasnau, R. \& Hartmann, L. (1983) Psychiatry in the Kingdom of Saudi Arabia. American Journal of Psychiatry, 140, 1493-1494.

$A$ full list of references is available on request from the author.

\title{
Links with child psychiatry in the USSR
}

\author{
Stewart Britten, Loughton Child Guidance Clinic, Essex
}

In 1988 the UK-USSR Medical Exchange Programme concluded an agreement with the Medical Association of the Union of Soviet Friendship Socie- ties on a rolling programme of exchange visits. The fields chosen for starting were mother and child health, with British specialists spending one to two 